\title{
Burst-Induced Synaptic Depression and Its Modulation Contribute to Information Transfer at Aplysia Sensorimotor Synapses: Empirical and Computational Analyses
}

\author{
Gregg A. Phares, ${ }^{1}$ Evangelos G. Antzoulatos, ${ }^{1}$ Douglas A. Baxter, ${ }^{2}$ and John H. Byrne ${ }^{1}$ \\ ${ }^{1}$ W. M. Keck Center for the Neurobiology of Learning and Memory, and ${ }^{2}$ Center for Computational Biomedicine, Department of Neurobiology and \\ Anatomy, The University of Texas-Houston Medical School, Houston, Texas 77030
}

The Aplysia sensorimotor synapse is a key site of plasticity for several simple forms of learning. Plasticity of this synapse has been extensively studied, albeit primarily with individual action potentials elicited at low frequencies. Yet, the mechanosensory neurons fire high-frequency bursts in response to even moderate tactile stimuli delivered to the skin. In the present study, we extend this analysis to show that sensory neurons also fire bursts in the range of $1-60 \mathrm{~Hz}$ in response to electrical stimuli similar to those used in behavioral studies of sensitization. Intracellular stimulation of sensory neurons to fire a burst of action potentials at $10 \mathrm{~Hz}$ for 1 sec led to significant homosynaptic depression of postsynaptic responses. The depression was transient and fully recovered within $10 \mathrm{~min}$. During the burst, the steady-state depressed phase of the postsynaptic response, which was only $20 \%$ of the initial EPSP of the burst, still contributed to firing the motor neuron. To explore the functional contribution of transient homosynaptic depression to the response of the motor neuron, computer simulations of the sensorimotor synapse with and without depression were compared. Depression allowed the motor neuron to produce graded responses over a wide range of presynaptic input strength. In addition, enhancement of synaptic transmission throughout a burst increased motor neuron output substantially more than did preferential enhancement of the initial phase of a burst. Thus, synaptic depression increased the dynamic range of the sensorimotor synapse and can, in principle, have a profound effect on information processing.

Key words: burst; depression; modulation; facilitation; synaptic filtering; synaptic plasticity

\section{Introduction}

The synapses of Aplysia mechanosensory neurons with the motor neurons that mediate reflexive withdrawal behaviors are important sites of plasticity underlying forms of learning including habituation, sensitization, and classical conditioning (Castellucci and Schacher, 1990; Byrne et al., 1991, 1993; Hawkins et al., 1993; Bailey et al., 1994; Byrne and Kandel, 1996; Kandel, 2001). Virtually all of the previous studies that analyzed the mechanisms of plasticity of the sensorimotor connections probed the synapse with a single presynaptic action potential or action potentials elicited at low frequency (e.g., interstimulus intervals between 1 and $600 \mathrm{sec}$ ). Aplysia sensory neurons can certainly fire single action potentials in response to a single weak, tactile stimulus to the skin, but they are more likely to fire a burst of spikes when the stimulus is sufficient to evoke a withdrawal behavior (Byrne et al., 1974, 1978a,b; Walters et al., 1983a; Clatworthy and Walters, 1993; Stopfer and Carew, 1996; Frost et al., 1997; Antonov et al., 1999, 2001, 2003).

Artificially induced bursts in Aplysia sensory neurons have

Received March 17, 2003; revised June 12, 2003; accepted June 25, 2003.

This work was supported by National Institutes of Health Grants P01-NS38310, R01-NS19895, and R01-RR11626. Correspondence should be addressed to Dr. John H. Byrne, Department of Neurobiology and Anatomy, The University of Texas-Houston Medical School, 6431 Fannin Street, MSB 7.046, Houston, TX 77030. E-mail: john.h.byrne@uth.tmc.edu.

Copyright $\odot 2003$ Society for Neuroscience $\quad$ 0270-6474/03/238392-10\$15.00/0 been used as a means to induce several forms of plasticity at sensorimotor synapses, including: homosynaptic depression (Eliot et al., 1994b; Jiang and Abrams, 1998; Zhao and Klein, 2002), post-tetanic potentiation (Walters and Byrne, 1984; Schacher et al., 1990; Eliot et al., 1994b; Bao et al., 1997; Schaffhausen et al., 2001), associative, activity-dependent plasticity (Hawkins et al., 1983; Walters and Byrne, 1983; Buonomano and Byrne, 1990; Eliot et al., 1994a; Bao et al., 1998; Antonov et al., 2001), long-term potentiation (Walters and Byrne, 1985; Lin and Glanzman, 1994), and the modulation of calcium influx through voltage-gated channels (Eliot et al., 1993). From the examples reported in the studies above, it is clear that sensorimotor synapses depress substantially during repetitive stimulation at high frequencies. Nevertheless, none of these studies focused on the plasticity of sensorimotor synapses during such a burst of activity.

The observations that sensory neurons fire bursts of spikes in response to physiological stimuli and that the EPSPs produced by those bursts depress rapidly, raise several important issues. First, how does the depression of the EPSPs contribute to the activation of the motor neuron and to the behavior? Are the later EPSPs in a burst so depressed that they make little or no contribution to the activity of the motor neuron? Second, during learning-related enhancement of synaptic efficacy, does facilitation of late EPSPs in a burst contribute to enhanced activation of the motor neuron? 
In the present study, we first examined the rates of discharge that sensory neurons can reach while encoding electrical stimuli similar to those used to evoke withdrawal behaviors in various training protocols. In addition, we examined the homosynaptic depression of sensorimotor synapses that occurred in response to a presynaptic burst of action potentials and found that the depressed EPSPs could still drive action potentials in a postsynaptic motor neuron. Simulations of a model network that contained two sensory neurons that synapse onto a single motor neuron suggested that depression allowed the motor neuron to produce graded responses over a wide range of presynaptic input strength. Furthermore, information transfer at sensorimotor synapses can be modulated differentially by two forms of plasticity that produced different degrees of facilitation of the depressed EPSPs during a burst.

\section{Materials and Methods}

General. Aplysia californica (75-350 gm) were obtained from Alacrity Marine Biological Specimens (Redondo Beach, CA) and Marinus (Long Beach, CA) and were individually caged and kept in an aquarium containing artificial seawater (ASW) at $\sim 15^{\circ} \mathrm{C}$ with a $12 \mathrm{hr}$ light/dark cycle. Animals were fed dried seaweed every other day. Three types of artificial seawater were used. Normal ASW contained (in mM): $\mathrm{NaCl}, 460 ; \mathrm{KCl}, 10$; $\mathrm{CaCl}_{2}, 11 ; \mathrm{MgCl}_{2}, 30 ; \mathrm{MgSO}_{4}, 20 ; \mathrm{NaHCO}_{3}, 2.5$; and HEPES, 10 ; $\mathrm{pH}$ 7.7. Low $\mathrm{Ca}^{2+} /$ high $\mathrm{Mg}^{2+}$ ASW contained (in $\mathrm{mM}$ ): $\mathrm{NaCl}, 400 ; \mathrm{KCl}, 10$; $\mathrm{CaCl}_{2}, 1 ; \mathrm{MgCl}_{2}, 80 ; \mathrm{MgSO}_{4}, 20 ; \mathrm{NaHCO}_{3}, 2.5$; and HEPES, 10; $\mathrm{pH}$ 7.7. High $\mathrm{Ca}^{2+} /$ high $\mathrm{Mg}^{2+}$ ASW contained (in $\mathrm{mM}$ ): $\mathrm{NaCl}, 386 ; \mathrm{KCl}, 8$; $\mathrm{CaCl}_{2}, 13.8 ; \mathrm{MgCl}_{2}, 90 ; \mathrm{MgSO}_{4}, 20 ; \mathrm{NaHCO}_{3}, 2.5$; and HEPES, $10 ; \mathrm{pH}$ 7.7. During all procedures, except the initial dissection of the reduced tail preparation (see below), the preparations were maintained at $\sim 15^{\circ} \mathrm{C}$. Tail sensory and motor neurons were identified based on their location, size, and electrical properties (Walters et al. 1983a).

Reduced tail experiment. Animals were anesthetized by placing them in ice-cold seawater for $\sim 15 \mathrm{~min}$. The anesthetized animal was then placed on a dissection tray. The dorsal surface of the animal faced upward. The animal was pinned down through the anterior tentacles and the parapodia. The left side of its posterior tail was implanted with a pair of 8-inchlong Teflon-coated silver wires, which were used to deliver stimuli to the tail. This type of electrode, its placement, and general method of implantation were similar to that used in our previous behavioral studies (Scholz and Byrne, 1987; Goldsmith and Byrne, 1993; Cleary et al., 1998; Wainwright et al., 2002).

A longitudinal incision from the head to the mantle cavity was made. All internal organs and central ganglia except for the left pleural-pedal were removed. The nerves leaving the left pleural-pedal ganglia were severed, except for the LP9, which innervates the left posterior tail (i.e., the site where the electrodes were implanted). The reduced preparation was transferred to a recording chamber, pinned down, and bathed in normal ASW. The ganglia were pinned down on a Sylgard-coated platform, which was isolated from the rest of the preparation by a Vaseline partition. To reduce synaptic activity during dissection, the medium bathing the ganglia was switched to low $\mathrm{Ca}^{2+} /$ high $\mathrm{Mg}^{2+}$ ASW, and the ganglia were desheathed. After desheathing, the bathing medium was switched back to normal ASW. The preparation was rested for $30 \mathrm{~min}$ before the onset of the experiment. The tail was stimulated through the implanted electrodes. The test stimulus consisted of a $200 \mathrm{msec}$, AC shock $(60 \mathrm{~Hz})$ provided by a variable transformer for which the output was gated by a relay. The output of the variable transformer passed through a $20 \mathrm{k} \Omega$ resistor to provide a quasi-constant current. The short circuit current that passed through this circuit for testing stimuli was $\sim 5$ $\mathrm{mA}$. The afferent action potentials were recorded from the soma of the sensory neuron with an intracellular electrode (10-15 $\mathrm{M} \Omega$ ) and using standard electrophysiological techniques.

Preparations of isolated ganglia. Before dissection, animals were anesthetized by injecting isotonic $\mathrm{MgCl}_{2}$ ( $50 \%$ body mass). Intact pleuralpedal ganglia were removed from the animal and were pinned to the Sylgard-coated floor of a recording chamber. To reduce synaptic trans- mission during dissection, ganglia were placed in low $\mathrm{Ca}^{2+} / \mathrm{high}_{\mathrm{Mg}}{ }^{2+}$ ASW. The connective tissue sheath was surgically removed from each ganglion. The bath was replaced with the appropriate ASW (see below), and ganglia were rested a minimum of $1 \mathrm{hr}$ before an experiment.

Synaptic depression. Recordings to characterize synaptic depression during bursts were conducted in high $\mathrm{Ca}^{2+} /$ high $\mathrm{Mg}^{2+}$ ASW. This ASW suppresses spontaneous and polysynaptic inputs without affecting the amplitude of evoked synaptic transmission (Trudeau and Castellucci, 1992). Sensory neurons in the pleural ganglion were impaled with a single electrode $(10-15 \mathrm{M} \Omega$ ) while a constant current of $-0.5 \mathrm{nA}$ was passed through the electrode to prevent an impalement discharge. Motor neurons in the pedal ganglion were impaled with two electrodes: one to record membrane potential, and another to inject current. The synapse was tested before beginning the experiment to determine the threshold for eliciting an action potential in the sensory neuron. For each set of pleural-pedal ganglia, data were collected from only one synapse. After testing the connection, the motor neuron was hyperpolarized to $-80 \mathrm{mV}$ throughout the remainder of the experiment to prevent the summated EPSP from triggering an action potential. After a $10 \mathrm{~min}$ recovery period, the sensory neuron was stimulated for $1 \mathrm{sec}$ at $10 \mathrm{~Hz}$ ( $10 \mathrm{msec}$ pulses, 100 msec interpulse interval) with a current that was $\sim 1.3 \times$ the threshold current necessary to elicit a spike. An interburst interval of 10 min was used to allow the synapse to recover from any homosynaptic plasticity that may have been evoked by the burst. Pilot studies found that the initial EPSPs did not depress with this interburst interval, which indicated that a $10 \mathrm{~min}$ interval was sufficient to recover from any burstinduced depression or post-tetanic potentiation (Eliot et al., 1993). Data were analyzed using custom software written using Hewlett-Packard Visual Engineering Environment (HPVEE) version 4.0 (Hewlett-Packard, Palo Alto, CA). The peak amplitude of the initial EPSP was measured from the baseline. To measure the amplitude of the second-tenth EPSPs, the decay of each EPSP was fit using the exponential regression object of HPVEE. The decay was extrapolated to the point under the peak of the next EPSP, and the difference between the peak amplitude and the extrapolated decay of the previous EPSP was measured. This method was validated using a previously developed simulation of the sensorimotor synapse (White et al., 1993; see below). A series of 10 simulations were performed in which a model sensory neuron was stimulated at $10 \mathrm{~Hz}$ to produce from 1 to 10 action potentials and the corresponding EPSPs in a model motor neuron. To measure the $n^{\text {th }}$ EPSP, the burst with $n$ EPSPs was superimposed over the burst with $n-1$ EPSPs, and the amplitude was measured from the peak of EPSP ${ }_{n}$ to the falling phase of the last EPSP of the burst with one less EPSP. When these values were compared with the EPSP amplitudes from the 10-EPSP burst that were measured by the analysis program, no differences were found between the two methods.

Sensorimotor synapses depress with repeated activation (Castellucci et al., 1970; Byrne, 1982; Walters et al., 1983a). At higher frequencies, the level of depression reaches a steady state (see Fig. 2) (see also Walters and Byrne, 1983, 1984; Buonomano and Byrne, 1990; Eliot et al., 1993, 1994a,b; Zhao and Klein, 2002). Synaptic depression was characterized after normalization of the amplitude of each EPSP within a burst to the first EPSP of that burst. These amplitudes were then fit with a regression curve using Origin 7.0 (Origin Lab Corp., Northampton, MA) and the following equation:

$$
E P S P_{\mathrm{t}}=A \times e^{-t / \tau}+\mathrm{EPSP}_{\text {steady-state }},
$$

where EPSP $\mathrm{t}_{\mathrm{t}}$ is the normalized amplitude of an EPSP at time $t, A$ is $1-$ EPSP $_{\text {steady-state }}, t$ is the time from peak amplitude of the first EPSP in the burst, $\tau$ is the time constant for the cumulative depression of EPSP amplitudes during the burst, and $\mathrm{EPSP}_{\text {steady-state }}$ is the amplitude of the steady-state depressed EPSPs.

Synaptic drive. Recordings to characterize the contribution of EPSPs to the generation of postsynaptic action potentials during bursts in sensory neurons were conducted in normal ASW. Two sensory neurons were found that synapsed onto the same motor neuron. At least one of these sensory neurons was required to elicit a spike in the motor neuron in response to a single test stimulus. Subsequently, the two sensory neurons were simultaneously stimulated at $10 \mathrm{~Hz}(10 \mathrm{msec}$ pulses, $100 \mathrm{msec}$ in- 
terpulse interval) with a current that was $\sim 1.3 \times$ the threshold for eliciting a spike. For each experiment, the initial burst duration was randomly set to $0.1 \mathrm{sec}$ ( 1 presynaptic spike), $0.5 \mathrm{sec}$ ( 5 presynaptic spikes), or $1 \mathrm{sec}$ (10 presynaptic spikes). Bursts with the other two durations were evoked in random order with an intertrial interval of $10 \mathrm{~min}$, which was sufficient to allow the synapse to recover from any homosynaptic plasticity evoked by the preceding burst (see above).

Simulations. The effects of homosynaptic depression and heterosynaptic facilitation on the output of the motor neuron were examined using a previously developed mathematical model of the tail-withdrawal circuit (White et al., 1993) and the neurosimulator, Simulator for Neural Networks and Action Potentials (Ziv et al., 1994; Hayes et al., 2003). The software and other files necessary to run these simulations are available at http://snnap.uth.tmc.edu/. In the model, voltage-dependent ionic conductances are described by Hodgkin-Huxley type equations, and timedependent synaptic conductances are described by second-order ordinary differential equations (Ziv et al., 1994). The tail withdrawal reflex circuit includes monosynaptic connections from sensory neurons to motor neurons as well as excitatory, inhibitory, and modulatory interneurons (Cleary and Byrne, 1993; White et al., 1993; Cleary et al., 1995; Xu et al., 1995). During the physiological experiments (see Figs. 2, 3), stimulation of sensory neurons at $10 \mathrm{~Hz}$ for $1 \mathrm{sec}$ did not appear sufficient to recruit these interneurons (Cleary and Byrne, 1993; White et al., 1993). Therefore, only the monosynaptic sensorimotor synapse was used for the simulations in the present study. As noted for each type of simulation (see below), a single model motor neuron was activated by either one or two model sensory neurons. Although some motor neurons may express more than one class of postsynaptic receptor at sensorimotor synapses (Dale and Kandel, 1993; Conrad et al., 1999; Antonov et al., 2003), the synaptic conductance was modeled with a single class of postsynaptic current with a reversal potential of $+30 \mathrm{mV}$ (i.e., AMPA-like). Synaptic depression was described by a simple, first-order decay in the availability of transmitter (SD) that depends on whether or not an action potential is present:

$\frac{d S D}{d t}= \begin{cases}\frac{-S D}{\tau_{d}} & \text { during a presynaptic action potential } \\ \frac{1-S D}{\tau_{r}} & \text { in the absence of a presynaptic action potential }\end{cases}$

where $\tau_{\mathrm{d}}$ is the time constant for depression during an action potential, and $\tau_{\mathrm{r}}$ is the time constant for the recovery from depression. This model was found to provide a reasonable approximation of the kinetics of depression during a burst (compare Figs. 2, 4A). The model was not intended to accurately reflect the underlying mechanisms, which are undoubtedly more complex. In the model, $\tau_{\mathrm{d}}$, was set to $4.7 \mathrm{msec}$ for control simulations and simulations with increased synaptic gain. For simulations of differential modulation, $\tau_{\mathrm{d}}$, was adjusted so that synapses would depress to a steady-state level equal to that of the unmodulated synapse. $\tau_{\mathrm{r}}$ was set to $700 \mathrm{msec}$ for all simulations. For nonplastic synapses, transmitter availability was clamped to $100 \%$. To compare depression during the simulated bursts under the different conditions (i.e., depressing vs nonplastic or control vs modulated), it was necessary to prevent the motor neuron from firing spikes. For these simulations, the model motor neuron was hyperpolarized to $-90 \mathrm{mV}$ by injection of negative current, and a single sensory neuron was stimulated to fire action potentials at 10 $\mathrm{Hz}$ for $1 \mathrm{sec}$. To compare differences in synaptic drive, motor neurons were left at rest $(-50 \mathrm{mV})$, and two sensory neurons were stimulated simultaneously to fire action potentials at $10 \mathrm{~Hz}$ for $1 \mathrm{sec}$. Excitatory interneurons, present in the original model circuit (White et al., 1993), were not included in these simulations.

\section{Results}

Sensory neurons fire bursts in response to electrical stimuli similar to those used in behavioral experiments

Sensory neurons fire bursts of action potentials when activated by mechanical stimuli applied to the skin (Byrne et al., 1974, 1978a,b; Walters et al., 1983a). One example from an earlier study of the response of a siphon sensory neuron to a series of con-
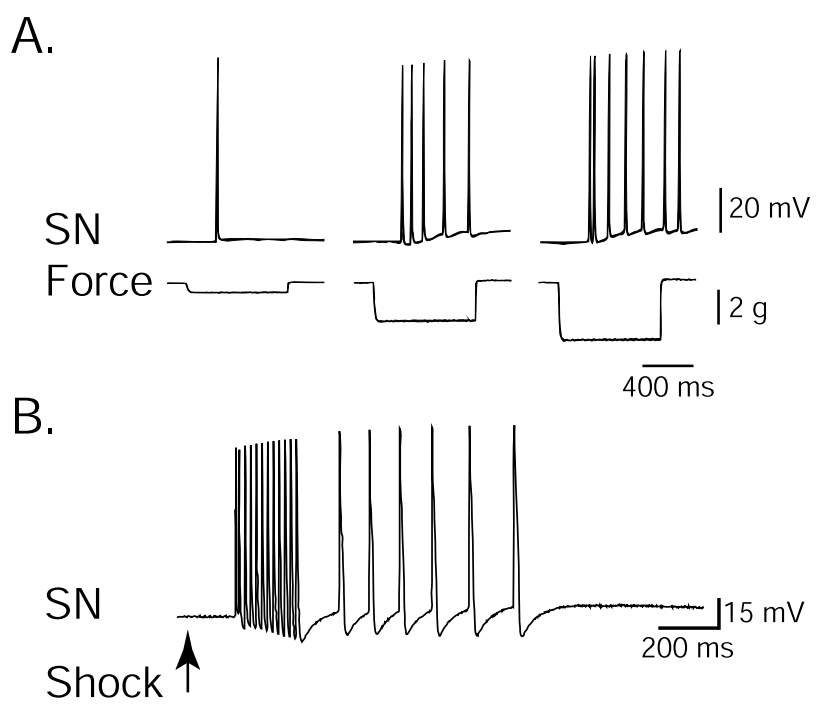

Figure 1. Responses of sensory neurons to mechanical and electrical stimulation of the skin. $A$, Sensory neurons fire bursts of spikes in response to moderate mechanical stimuli. Intracellular recordings of the response of a siphon sensory neuron to $800 \mathrm{msec}$ step forces delivered to the skin. The cellular discharge monotonically increases as a function of the stimulus intensity. Adapted from Byrne et al. (1978a). B, The response of a tail sensory neuron to a 200 msec shock to the tail (arrow) in a semi-intact preparation. The sensory neuron fires at a frequency that matches the stimulus $(\sim 60 \mathrm{~Hz})$ with little adaptation. This initial, time-locked discharge is followed by an afterdischarge during which the firing rate averages $\sim 10 \mathrm{~Hz}$.

trolled force stimuli to the skin is reproduced in Figure $1 \mathrm{~A}$ (adapted from Byrne et al., 1978a). Weak stimuli elicited a single spike, but even stimuli of intermediate intensity elicited a burst of spikes in the $10 \mathrm{~Hz}$ range. However, behavioral sensitization of withdrawal reflexes has been studied predominantly using electrical stimuli. Therefore, a key question is whether sensory neurons fire a burst of spikes under conditions similar to those used in behavioral studies, and if so, at what frequency? Figure $1 B$ illustrates the response of a tail sensory neuron to electrical stimulation of the tail in a reduced preparation. The stimulus duration used in this study $(200 \mathrm{msec})$ was within the range of durations $(20-500 \mathrm{msec})$ commonly used in behavioral studies of long-term sensitization (Scholz and Byrne, 1987; Goldsmith and Byrne, 1993; Cleary et al., 1998; Sutton et al., 2001; Wainwright et al., 2002). The brief electrical stimulus to the tail produced a prolonged burst of spikes in the sensory neuron (Fig. $1 B$ ). Initially, sensory neurons fired at a frequency that matched that of the stimulus (i.e., $60 \mathrm{~Hz}$ ). Subsequently, the neuron settled to a lower frequency of spiking during a period that outlasted the stimulus. In the example illustrated (Fig. $1 B$ ), the afterdischarge had an average frequency of $\sim 10 \mathrm{~Hz}$ and duration of $\sim 1 \mathrm{sec}$. The average frequency of the afterdischarge was $3.6 \pm 1.0 \mathrm{~Hz}(n=10$; range $1.2-10.3 \mathrm{~Hz}$ ), and the average duration was $3.3 \pm 1.2 \mathrm{sec}$ $(n=10$; range $0.5-6.5 \mathrm{sec})$. These results indicate the physiological relevance of bursts in the sensory neurons, and therefore, the importance of probing synaptic transmission with a burst of spikes.

\section{Synaptic efficacy depresses during bursts}

Although it is clear from published examples of bursts that sensorimotor synapses depress at frequencies of $>1 \mathrm{~Hz}$ (Buonomano and Byrne, 1990; Eliot et al., 1993, 1994a,b; Zhao and Klein, 2002), this depression has not been quantified. Figure $2 A$ shows an example of the postsynaptic response in a tail motor neuron 
A.
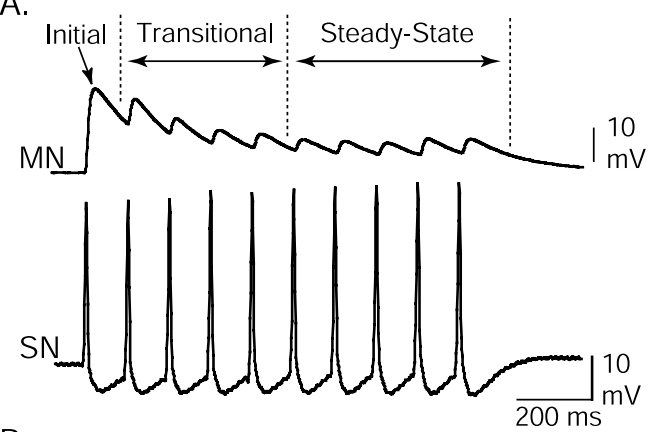

B.

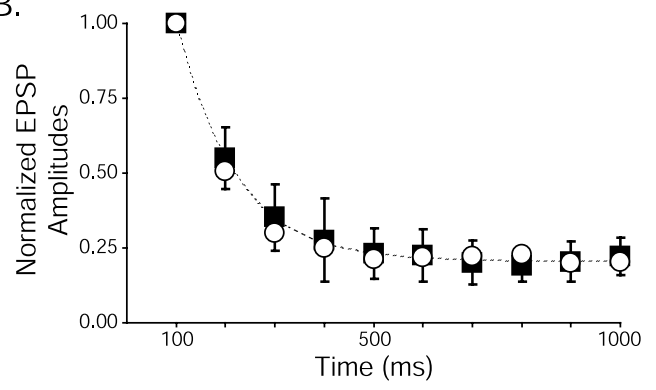

Figure 2. Synaptic depression during bursts. A, An example of a $10 \mathrm{~Hz}$, 1 sec burst of action potentials evoked in a pleural sensory neuron (SN) and the resulting summated EPSP recorded in a pedal motor neuron (MN). The amplitudes of individual EPSPs depress to a steady-state level of $\sim 20 \%$ of the initial EPSP. For analysis, the summated EPSP is divided into three phases: the initial EPSP, the transitional phase during which EPSP amplitude depressed (the secondfifth EPSPS), and the steady-state phase during which the level of depression was stable (the sixth to tenth EPSPs). B, Cumulative data for depression of sensorimotor synapses during $10 \mathrm{~Hz}$ bursts (filled squares; $n=10$ sensorimotor synapses from 10 preparations). EPSPs were normalized to the initial EPSP of each burst. The symbols represent EPSP amplitudes and are presented as means $\pm S D$ s. Open circles represent values of normalized EPSPs obtained from a simulation of a burst at a model sensorimotor synapse (i.e., EPSPs in Fig. $4 \mathrm{~A}$ ). The curve is the fit of the empirical data with the equation: PPSP $_{t}=0.80 \times e^{-t / 120.0}+0.20$.

during a $10 \mathrm{~Hz}, 1 \mathrm{sec}$ burst of action potentials evoked in a tail sensory neuron. The postsynaptic response was divided into three phases: the initial EPSP, the transitional phase (second to fifth EPSP) during which synaptic efficacy decreased, and the steady-state phase (sixth to tenth EPSP), during which EPSP amplitudes reached a stable level of depression. Figure $2 B$ illustrates cumulative data for $10 \mathrm{~Hz}$ bursts in which the EPSP amplitudes were normalized to the amplitude of the initial EPSP of the burst ( $n=10$ sensorimotor synapses in 10 pleural-pedal ganglia). Sensorimotor synapses depressed to an average steady-state level of $20.0 \pm 4.7 \%$ with a time constant of $120.0 \pm 39.5 \mathrm{msec}$. Given the fairly substantial depression of the EPSPs during a burst, the question arises as to what extent, if any, the depressed EPSPs contribute to the firing of the motor neuron.

\section{Contribution of steady-state depressed EPSPs to postsynaptic spike activity}

To determine whether the steady-state synaptic response contributes to the synaptic drive of the motor neuron, we examined the ability of presynaptic spikes during a burst to elicit postsynaptic spike activity. Therefore, in each of eight preparations, two tail sensory neurons were activated simultaneously, while recording from a single motor neuron that received convergent input from the sensory neurons. Each preparation was tested with trains of 1,5 , and 10 spikes elicited simultaneously in two sensory neurons (intertrial interval $=10 \mathrm{~min}$ ). For trains with more than one spike in each sensory neuron, sensory neurons were stimulated to fire at $10 \mathrm{~Hz}$. All motor neurons fired a single action

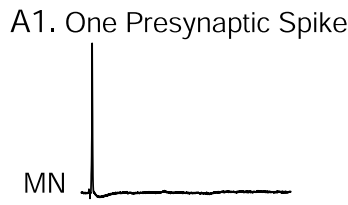

B1. Five Presynaptic Spikes

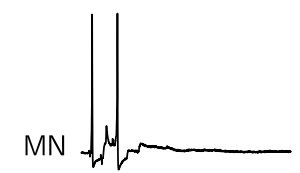

C1. Ten Presynaptic Spikes
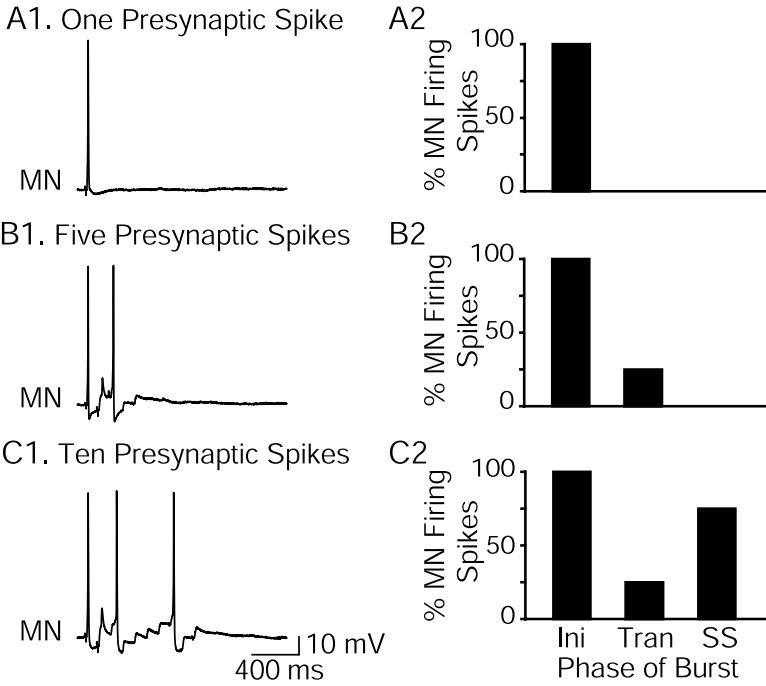

B2

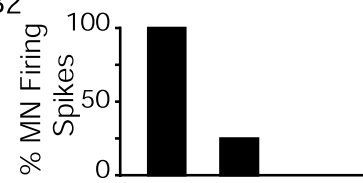

$\mathrm{C} 2$

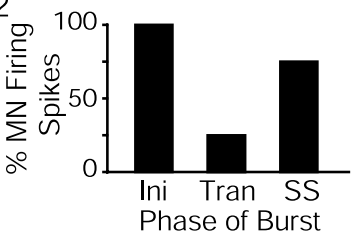

Figure 3. The steady-state phase of the synaptic response contributes to the synaptically driven spike activity in motor neurons. Two sensory neurons were simultaneously stimulated to elicit spikes (data not shown), and the number of resulting spikes in a postsynaptic motor neuron (MN) was recorded. The effects of three types of presynaptic activity were examined: a single spike in each sensory neuron, 5 spikes at $10 \mathrm{~Hz}$ in each sensory neuron, and 10 spikes at 10 $\mathrm{Hz}$ in each sensory neuron. $A$, Response of motor neurons to a single spike in each sensory neuron. $A 1$, Example trace from a motor neuron. $A 2$, Cumulative data for eight preparations illustrating the percentage of motor neurons that fired at least one spike during each phase of the synaptic response. The initial phase (Ini) includes only the spikes elicited by the first EPSP. The transitional phase (Tran) refers to the time period corresponding to the second through the fifth EPSPS, and the steady-state phase (SS) refers to the time period corresponding to the sixth through the tenth EPSPs. B, Response of motor neurons when pairs of sensory neurons were stimulated to fire five spikes at $10 \mathrm{~Hz}$. C, Response of motor neurons when pairs of sensory neurons were stimulated to fire 10 spikes at $10 \mathrm{~Hz}$. The same motor neuron was illustrated in $A 1$, $B 1$, and $C 1$.

potential in response to a single, simultaneous pair of presynaptic spikes (Fig. 3A). When four additional presynaptic spikes were evoked in each sensory neuron, only $25 \%$ of the motor neurons fired action potentials during the transitional phase of the postsynaptic response (Fig. 3B). However, when five more presynaptic spikes were included in the presynaptic train, $75 \%$ of the motor neurons fired an additional action potential during the steady-state phase of the synaptic response (Fig. 3C). Despite the fact that the steady-state phase EPSPs were depressed to $\sim 20 \%$ of the initial EPSP (Fig. 2), they still contributed to driving spike activity in the motor neuron. Because the magnitude of a withdrawal response is proportional to the number of spikes in the motor neuron (Walters et al., 1983b), the increased drive provided by an extended presynaptic burst over that of a single spike would be expected to have a substantial impact on amplitude and duration of the behavioral response.

Contribution of synaptic depression to motor neuron output To determine the contribution that synaptic depression makes to the firing pattern of the motor neuron, we took advantage of a previously developed mathematical model of the tail-withdrawal circuit (White et al., 1993). The original model was constructed without including properties of synaptic depression into sensorimotor synapses. Figure $4 \mathrm{~A}$ illustrates example simulations for both nonplastic (gray curve) and depressing synapses (black curve). During these simulations, the motor neuron was hyperpolarized to $-90 \mathrm{mV}$ to prevent it from firing action potentials, and a single sensory neuron was used to produce the synaptic response. Next, a second sensory neuron with identical properties was added to the circuit, and the simulation was repeated 
A. Depressing and Nondepressing EPSPS

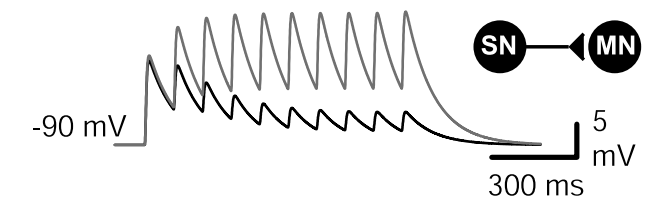

B1. Response to Nondepressing EPSPs

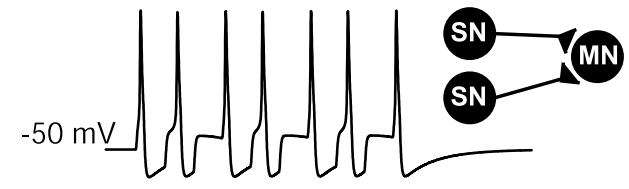

B2. Response to Depressing EPSPs

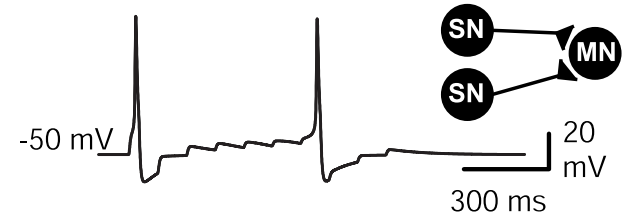

Figure 4. Contribution of synaptic depression to information transfer at simulated sensorimotor synapses. Details of the model are described by White et al. (1993). In the present study, only two sensory neurons and a motor neuron were simulated, and the ability to simulate homosynaptic depression was included. A, Summated EPSPs in a model motor neuron hyperpolarized to $-90 \mathrm{mV}$ to prevent spiking. One of the model sensory neurons (data not shown) was stimulated to fire a $10 \mathrm{~Hz}, 1$ sec burst of action potentials. The gray trace represents a model synapse that did not include plasticity, whereas the black trace represents a model synapse that included depression, which was adjusted to closely mimic empirical data (Fig. 2). Note that the amplitude of the initial EPSP was identical in both simulations. B, Synaptic depression greatly reduced the number of motor neuron action potentials elicited by bursts evoked simultaneously in two model sensory neurons. For these simulations, the resting potential of the motor neuron was $-50 \mathrm{mV}$, which allowed it to fire action potentials. $B 1$, The response of the motor neuron to the EPSPs that did not depress. B2, The response of the motor neuron to the EPSPs that exhibited synaptic depression. On the far right is a schematic diagram of the circuit used for each simulation.

with the motor neuron at its resting membrane potential $(-50$ $\mathrm{mV}$, approximately the resting potential recorded for tail motor neurons in isolated ganglia) to examine the extent to which the synaptic input could fire the motor neuron (Fig. 4B1). Under these conditions, simultaneous activity in the two sensory neurons at $10 \mathrm{~Hz}$ for $1 \mathrm{sec}$ elicited seven spikes in the motor neuron (Fig. 4 B1). Next, the original model of White et al. (1993) was modified to simulate the magnitude and kinetics of synaptic depression that were observed empirically (Fig. 2). Within the simulated synaptic response, the tenth EPSP was $\sim 20 \%$ of the initial EPSP (Fig. 4A, black curve). There is also a good match for the transitional phase EPSPs (Fig. 2C, open circles), showing that the model synapse depressed with kinetics similar to those recorded in the ganglion. Thus, there was a good match between the empirical and modeling results. Now when the two sensory neurons with plastic synapses were fired simultaneously, only two spikes were elicited in the motor neuron (Fig. 4 B2) in a pattern that was similar to that observed empirically (Fig. 3C). The last postsynaptic spike occurred during the steady-state phase of the synaptic response.

We also examined the pattern of activity in the motor neuron when the sensory neuron was activated in a pattern that mimicked its response to tactile stimulation of the skin (i.e., empirical data of Fig. 1A). Figure 5 illustrates the simulated response to moderate and strong intensity stimuli. The pattern of the motor neuron output was essentially identical to that in response to the $10 \mathrm{~Hz}$ burst. Specifically, the motor neuron fired two spikes: one
A. Moderate Stimulus

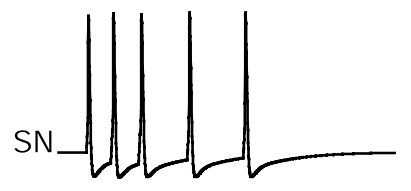

B. Strong Stimulus

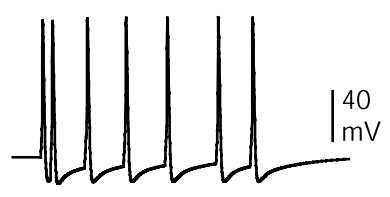

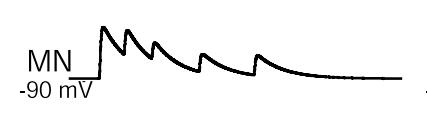
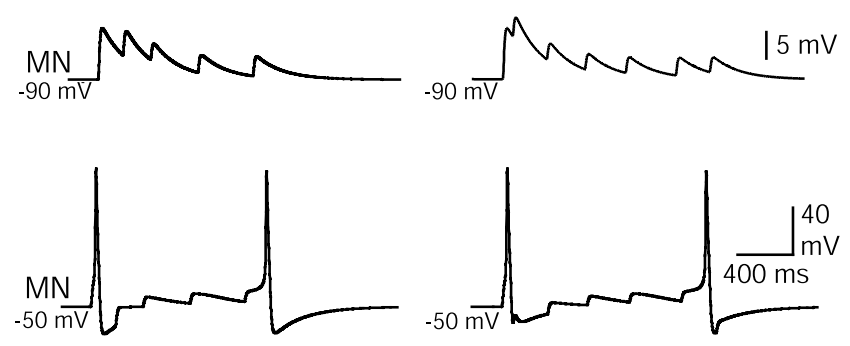

Figure 5. Simulated response to mechanical stimuli. $A$, Model sensory neurons were stimulated in a pattern similar to those shown in Figure $1 A$ (middle) to mimic a tactile stimulus of moderate intensity. $B$, Model sensory neurons were stimulated in a pattern like those shown in Figure $1 A$ (right) to mimic a strong tactile stimulus. Top, Spike pattern of a model sensory neuron. Middle, EPSPs recorded in a motor neuron in response to stimulation of a single sensory neuron with the pattern above. The motor neuron was hyperpolarized to $-90 \mathrm{mV}$ to reduce the likelihood of action potentials being generated. Bottom, Pattern of spikes generated in a motor neuron by stimulation of two sensory neurons with the pattern above. The synaptic conductance and kinetics of depression are the same as described for depressing synapse in Figure 4.

in response to the initial EPSP and the second late in the burst in response to a depressed EPSP. Therefore, the results illustrated in Figures 4 and 5 indicate that the presence of transient homosynaptic depression at the sensorimotor synapse had an important influence on information transfer in this simple neural circuit. Specifically, homosynaptic depression acted like a filter and decreased the fidelity with which the pattern of activity in the sensory neuron was represented by action potentials in the motor neuron. The filtering properties of synaptic depression have been discussed previously (Castro-Alamancos, 1997; Bertram, 2001; Fortune and Rose, 2001; Izhikevich et al., 2003).

\section{Contribution of heterosynaptic facilitation to motor neuron output}

Changes in the efficacy of transmission at sensorimotor synapses are thought to be important for several forms of learning (Byrne et al., 1991; Carew, 2000; Hawkins et al., 1993; Byrne and Kandel, 1996; Bailey et al., 2000). How might different modulatory actions influence the firing response of the motor neuron? To examine this issue, we simulated the effects of two forms of plasticity during bursts: a uniform increase in the gain of the synapse and differential modulation of synaptic efficacy that preferentially increased synaptic efficacy in the earlier phases of the burst. We focused on these two types of modulation because the sensory neurons are modulated in such a manner by serotonin and small cardioactive peptide B, respectively (Phares and Byrne, 1999). Specifically, serotonin produces a gain increase at sensorimotor synapses, whereas small cardioactive peptide B facilitates predominantly the first few EPSPs of a burst (Phares and Byrne, 1999). The uniform gain increase was modeled by doubling the magnitude of the synaptic conductance of the sensorimotor synapse, whereas the rate of homosynaptic depression remained unchanged (Fig. 6A). Under these conditions, the same burst of spikes in the two sensory neurons now elicited four spikes in the motor neuron (Fig. 6 B2), and two of the spikes occurred during 

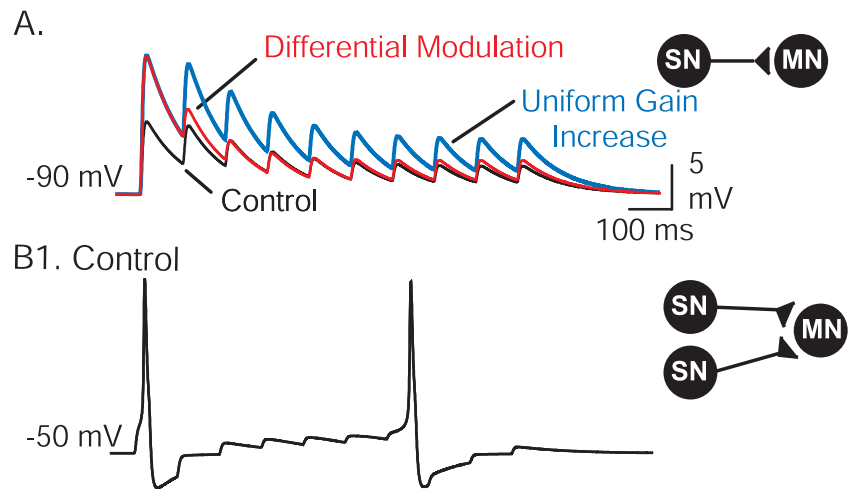

B2. Uniform Gain Increase

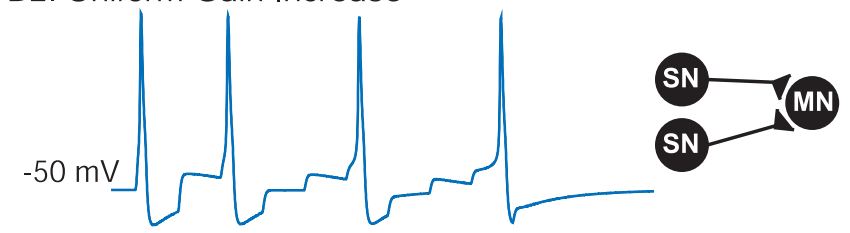

B3. Differential Modulation

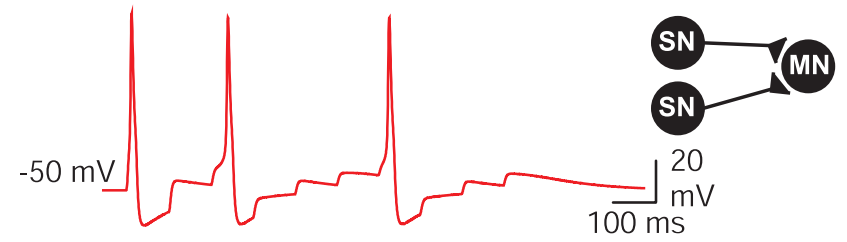

Figure 6. Effects of different types of heterosynaptic modulation at simulated sensorimotor synapses. $A$, Simulated synaptic responses in a motor neuron evoked by a $10 \mathrm{~Hz}, 1$ sec burst of spikes in one sensory neuron. The motor neuron was held at $-90 \mathrm{mV}$ to prevent spiking. Control (black line) represents the postsynaptic response of the unmodulated sensorimotor synapse. For "uniform gain increase" (blue line) the synaptic conductance was doubled without changing the kinetics of depression. Thus, the amplitude of each EPSP was increased compared to the control. For "differential modulation" (red line) the conductance was doubled, and the kinetics of depression were made faster $\left(\tau_{\mathrm{d}}=2 \mathrm{msec}\right.$ ). Note that the amplitudes of the final EPSPs were the same as those in the control trace. $B$, Simulated responses of the motor neuron to a $10 \mathrm{~Hz}, 1 \mathrm{sec}$ burst of spikes in two sensory neurons under the three conditions described in A. For these simulations, the resting potential of the motor neuron was $-50 \mathrm{mV}$, which allowed it to fire action potentials. Colors represent the same conditions as in A. On the far right is a schematic diagram of the circuit used for each simulation.

the steady-state phase of the synaptic response. Thus, a uniform increase in the gain increased the fidelity of information transfer (i.e., decreased the filtering properties) of the synapse.

To simulate the effects of differential modulation of synaptic efficacy, the magnitude of the synaptic conductance was doubled (as previously), and the rate of homosynaptic depression was increased such that the tenth EPSP was approximately the same amplitude as the last EPSP of the control burst (Fig. 6A). In this case, the sensory neuron burst only elicited three postsynaptic spikes, only one of which occurred during the steady-state phase (Fig. 6B3). Thus, in principle, differential facilitation would produce a smaller change in the magnitude and duration of a withdrawal behavior than would a uniform increase in synaptic gain. To test this hypothesis, a series of simulations was performed in which the initial EPSP was increased to five times that of the basal EPSP. As the initial EPSP was increased, $\tau_{\mathrm{d}}$ was also increased to maintain the amplitude of the steady-state phase EPSPs at basal values. Even with this large increase in the amplitude of the initial EPSP, only one additional spike was triggered in the motor neuron (three spikes in the initial and transitional phases, and one spike during the steady-state phase; data not shown). These data illustrate that differential modulation of the early phases of the burst and a uniform increase in synaptic gain (see also below) resulted in motor neuron spike patterns that differed both qualitatively and quantitatively. Because both frequency and duration of the motor neuron burst determine the amplitude of muscle contraction (McPherson and Blankenship, 1991), an increase in the gain of the sensorimotor synapse should be more effective in driving contractions than differential modulation for the same level of facilitation of the initial EPSP.

A third form of plasticity was also examined. Jiang and Abrams (1998) reported a transient form of paired-pulse facilitation at abdominal ganglion sensorimotor synapses. This form of homosynaptic facilitation was seen only when sensory neurons were activated at frequencies $>1 \mathrm{~Hz}$ and only for the first burst tested. To simulate such a condition during a $10 \mathrm{~Hz}, 1 \mathrm{sec}$ burst, the initial EPSP and steady-state EPSPs were not modified, but the transitional phase EPSPs were facilitated. The second EPSP was twice the amplitude of the first. In response to this type of plasticity, the motor neuron fired three spikes, all of which occurred during the initial and transitional phases of the burst (data not shown). These simulations showed that only a single additional spike was generated by the motor neuron in response to increased efficacy during the transitional phase of the burst. Thus, this second example of differential modulation emphasized the onset of the burst, but was less effective at driving spikes in the motor neuron late in the burst.

\section{Depression increases the dynamic range of sensorimotor synapses}

Although homosynaptic depression decreased the fidelity of eliciting a postsynaptic spike, our simulation studies suggest that depression may also increase the dynamic range of the postsynaptic response. Two series of simulations were performed in which the synaptic gains were progressively increased to represent increasing levels of modulation. In one series, the sensorimotor synapses depressed (Fig. 7A), whereas in the other series, they did not (Fig. $7 B$ ). In these simulations, postsynaptic responses at nondepressing synapses were saturated with only a threefold increase in gain (Fig. 7B3), whereas the postsynaptic responses at depressing synapses displayed a greater dynamic range (Fig. 7C). This larger dynamic range in the postsynaptic response may also be important because cutaneous stimuli often activate multiple (e.g., 4-8) sensory neurons (Byrne et al., 1974, 1978a; Walters et al., 1983a). As the number of active presynaptic neurons increases, the postsynaptic response would saturate, and learning-related increases in synaptic efficacy would have little effect. Thus, the larger dynamic range that emerged from homosynaptic depression may be critical if the learning-induced increases in the gain of the synapse are to have any substantial effect.

\section{Discussion}

Aplysia mechanosensory neurons fire bursts of action potentials in response to even moderate mechanical stimuli (Byrne et al., 1974, 1978a,b; Walters et al., 1983a). The data presented here (Fig. $1 B$ ) show that mechanosensory neurons also fire bursts when activated by electrical stimuli of the type that are commonly used in studies of learning (Scholz and Byrne, 1987; Goldsmith and Byrne, 1993; Stopfer and Carew, 1996; Cleary et al., 1998; Sutton et al., 2001; Wainwright et al., 2002). Data from two previous studies illustrated that electrical stimuli delivered to the tail evoke bursts of action potentials in sensory neurons (Clatworthy and Walters, 1993; Stopfer and Carew, 1996). The evoked bursts 


\section{Depressing Nondepressing}
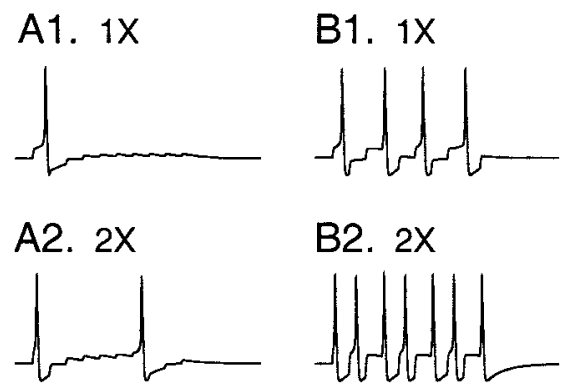

B2. $2 X$

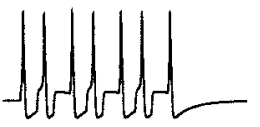

A3. $3 X$

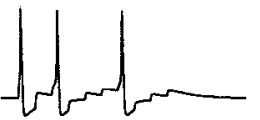

B3. $3 X$

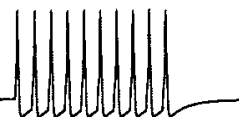

A4. $4 \mathrm{X}$

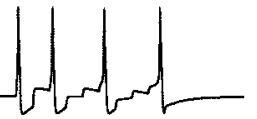

B4. $4 \mathrm{X}$

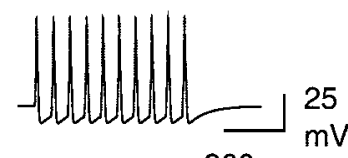

$300 \mathrm{~ms}$

C.

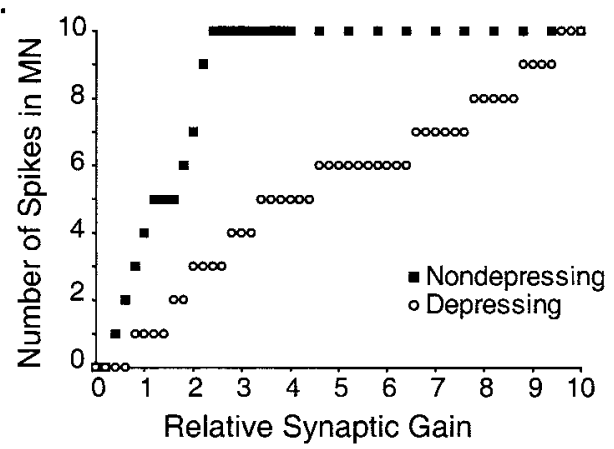

Figure 7. Synaptic depression increases the dynamic range of the postsynaptic response. Simulations of a model sensorimotor synapse with or without depression. These simulations used only a single sensory neuron. Synaptic gain, representing heterosynaptic modulation, was increased uniformly from 1 to $10 \times$. $A$, Depressing synapses had a wide dynamic range. Increasing gain from 1 to $4 \times$ gradually leads to more postsynaptic spikes. $B$, Over the same range of synaptic gain, nondepressing synapses had a narrow dynamic range. C, Histogram showing the number of spikes generated at simulated sensorimotor synapses as the gain was varied. Open circles represent a depressing synapse as shown in $A$. Filled squares represent a nondepressing synapse as in $B$. Increasing gain led to an increase in the number of postsynaptic spikes that saturated at $<3 \times$ for the nondepressing synapse, but not until $>9 \times$ for the depressing synapse.

were approximately the same duration (100-400 msec) and frequency $(20-60 \mathrm{~Hz})$ as the electrical stimuli. Although these studies found that electrical stimuli elicit bursts, the intensity of the stimuli were either weaker (i.e., at the threshold for withdrawal in Stopfer and Carew, 1996) or stronger (i.e., sufficient to mimic the behavioral response to a strong tail pinch with toothed forceps in Clatworthy and Walters, 1993) than those typically used as test stimuli in learning protocols in intact animals (Goldsmith and Byrne, 1993; Cleary et al., 1998; Wainwright et al., 2002). The results presented here used moderate strength electrical stimuli. These stimuli evoked biphasic bursts in sensory neurons. In the initial phase, the frequency of sensory neuron spikes was essentially locked to that of the AC stimulus (Fig. $1 B$ ). The second phase had a lower frequency and resembled the afterdischarge reported by Clatworthy and Walters (1993; Byrne et al. 1974). Together, these results support the idea that the study of synaptic transmission and plasticity during bursts is important because bursts are the predominant mode of transmission at sensorimotor synapses in behavioral paradigms used to study learning and memory in this model system.

\section{Depression at sensorimotor synapses}

Most previous studies examined depression by eliciting individual spikes at low frequencies. Even at $0.01 \mathrm{~Hz}$, synapses of LE sensory neurons onto motor neuron L7 in the abdominal ganglion depress to $65 \%$ of the initial EPSP $\left(\right.$ EPSP $\left._{1}\right)$ by the tenth EPSP (EPSP 10 ) (Byrne, 1982). As the frequency increases, so does the magnitude of the depression. At $1 \mathrm{~Hz}, \mathrm{EPSP}_{10}$ is depressed to $30-36 \%$ of $\mathrm{EPSP}_{1}$ (Byrne, 1982; Eliot et al., 1994b). At frequencies between 0.01 and $1 \mathrm{~Hz}$, the relationship of depression and frequency appears to be rather complex (Byrne, 1982; Eliot et al., 1994b), and depression at these low frequencies may involve multiple mechanisms (Zhao and Klein, 2002). The functional role of this low frequency depression is unknown, but it is believed to contribute to habituation of the withdrawal reflex (Castellucci et al., 1970; Kupfermann et al., 1970; Byrne, 1982; Cohen et al., 1997; Frost et al., 1997).

Few studies have examined depression at sensorimotor synapses during brief, high-frequency bursts. At cultured somasoma synapses stimulated at $25 \mathrm{~Hz}$ for $400 \mathrm{msec}$, sensorimotor synapses depress to $\sim 24 \%$ of the initial EPSP and reach a steadystate level of depression by the third spike (Zhao and Klein, 2002). This value is similar to that reported in the present study. During a $10 \mathrm{~Hz}, 1 \mathrm{sec}$ burst, synapses depressed to a steady-state level of $\sim 20 \%$ of the initial EPSP (Fig. 2). This burst-induced homosynaptic depression appeared to reduce the fidelity with which the pattern of spikes in the postsynaptic motor neuron (output) duplicates the pattern of spikes of the presynaptic sensory neuron (input).

\section{Modulation of synaptic efficacy during bursts}

The effects of heterosynaptic modulation were considered with respect to their interaction with homosynaptic depression using several forms of synaptic facilitation. The first class was a uniform increase in the gain of a synapse. A gain increase occurs when heterosynaptic plasticity enhances synaptic efficacy but does not alter the degree of homosynaptic plasticity. Increased synaptic gain (Fig. 6A,B2) may allow sensory neurons to more effectively drive motor neuron activity, and thus, more effectively evoke the behavior. This type of modulation occurs at sensorimotor synapses in response to serotonin (Phares and Byrne, 1999) and is induced by long-term potentiation in the CA1 region of the mammalian hippocampus (Buonomano, 1999; Selig et al., 1999). The second class occurs when heterosynaptic plasticity differentially modulates synaptic efficacy during a train of spikes. Differential modulation may, for example, only increase synaptic efficacy at the beginning of a train of spikes (Fig. 6A,B3), thereby enhancing the response to stimulus onset. At sensorimotor synapses, this type of modulation occurs in response to small cardioactive peptide B (Phares and Byrne, 1999). Differential modulation is also observed in mammalian neocortex after induction of long-term potentiation (Markram and Tsodyks, 1996; Tsodyks and Markram, 1997; Buonomano, 1999). The present study illustrated that these two classes of facilitation are likely to have dif- 
ferent effects on information transfer. A uniform increase in the synaptic gain increased the fidelity with which the pattern of spikes in the motor neuron more closely matched that of the sensory neuron input (Figs. 6B2, 7). In contrast, differential modulation produced a pattern of postsynaptic activity that better represents the initial phase of the input (Fig. 6B3).

Other forms of plasticity are of course possible. For example, when sensorimotor synapses are initially stimulated to fire bursts with frequencies $>1 \mathrm{~Hz}$, they may exhibit a transient form of paired-pulse facilitation (Jiang and Abrams, 1998). This form of homosynaptic plasticity effects only the transitional phase of the burst and dissipates rapidly with repeated activation of the synapse. Despite the large enhancement of synaptic efficacy during the transitional phase, simulations showed that only a single additional action potential is generated by the motor neuron. However, the motor neuron output was shifted to the first half of the presynaptic burst. Future studies may find that other forms of modulation alter the efficacy of sensorimotor synapses in ways that have not been considered here. For example, some form of plasticity may only affect transmission during the steady-state phase of the burst.

\section{The role of synaptic depression in information transfer}

Depression can play several different roles in information processing and transfer. For example, depression has been suggested to play an important role in balancing the relative gain of lowand high-frequency inputs (Abbott et al., 1997), to convert temporal signals into a spatial code (Buonomano and Merzenich, 1995; Buonomano et al., 1997), and to regulate bistability of central pattern generators (Nadim et al., 1999; Manor and Nadim, 2001). The role played by depression in filtering information transfer at synapses depends in part on the mechanisms that produce it (Bertram, 2001; Bertram et al., 2002).

Although the mechanisms of high-frequency depression at sensorimotor synapses are poorly understood, depletion of synaptic vesicles appears to make an important contribution (Zhao and Klein, 2002). Thus, the sensorimotor synapse may act as a filter of afferent activity. Indeed, when the simulated monosynaptic circuit consisted of nondepressing sensorimotor synapses, the burst of 10 presynaptic spikes produced seven EPSPs with absolute amplitudes sufficient to produce postsynaptic spikes (Fig. 4B1). This number was substantially reduced when depression was included in the simulation (Fig. 4B2). Therefore, depression reduced the information about the stimulus that was conveyed for any given submaximal synaptic strength. Yet, despite the level of depression reached during bursts of action potentials, steady-state EPSPs were still able to elicit action potentials in motor neurons (Fig. 3). As the gain of the synapse increased, even the depressing synapse recruited more postsynaptic action potentials. Eventually, a ratio of 1:1 was attained (Fig. $7 C)$. What sensory information is transferred to the motor neuron? If only the presynaptic activity is considered, it would appear that sensory neurons encode the stimulus strength in the frequency and duration of their afferent burst (Byrne et al., 1974, 1978a). However, if homosynaptic depression at higher frequencies is frequency-dependent (as it is at lower frequencies), then more intense stimuli should lead to more rapid depression at sensorimotor synapses, and consequently, to less drive of motor neuron spiking activity. Both the empirical data and simulations presented here suggest that, at synapses unmodified by heterosynaptic modulation, sensory neurons signal the onset of the stimulus to the motor neuron, providing the initial depolarization to trigger motor neuron activity. Moreover, the sensory neu- rons also appeared to signal aspects of the duration of the stimulus. Caused in part by synaptic depression, as well as by the magnitude and duration of the afterhyperpolarization, motor neurons preferentially fired action potentials at the beginning and end of presynaptic bursts (Figs. 3, 4)

Depression may also serve to increase the dynamic range of sensorimotor synapses. Each motor neuron receives inputs from multiple sensory neurons (Byrne et al., 1974; Walters et al., 1983a). Without synaptic depression, information about stimulus onset and duration conveyed by multiple, simultaneously responding sensory neurons would saturate with the recruitment of very few sensory neurons (Fig. 7). Under this condition, there would be little room for heterosynaptic facilitation to modify the output of the motor neuron.

Irrespective of which mechanisms lead to activity-dependent plasticity, the present study underscores the importance of probing synapses with physiological patterns of activity. Sensory neurons fire bursts in response to either electrical or mechanical stimuli that are sufficient to evoke withdrawal responses. These bursts produce strongly depressing postsynaptic responses that limit the information transfer across the synapse. The depressing responses may also increase the dynamic range of the response that is available for increased synaptic gain because of modulation of individual synapses, or recruitment of additional sensory neuron inputs, or both of these mechanisms acting in concert. Further characterization of synaptic transmission and its modulation during bursts will likely provide new insights into the cellular mechanisms that underlie neuronal plasticity and the modification of behavior.

\section{References}

Abbott LF, Varela JA, Sen K, Nelson SB (1997) Synaptic depression and cortical gain control. Science 275:220-224.

Antonov I, Kandel ER, Hawkins ER (1999) The contribution of facilitation of monosynaptic PSPs to dishabituation and sensitization of the Aplysia siphon withdrawal reflex. J Neurosci 19:10438-10450.

Antonov I, Antonova I, Kandel ER, Hawkins RD (2001) The contribution of activity-dependent synaptic plasticity to classical conditioning in Aplysia. J Neurosci 21:6413-6422.

Antonov I, Antonova I, Kandel ER, Hawkins RD (2003) Activity-dependent presynaptic facilitation and Hebbian LTP are both required and interact during classical conditioning in Aplysia. Neuron 37:135-147.

Bailey CH, Alberini C, Ghirardi M, Kandel ER (1994) Molecular and structural changes underlying long-term memory storage in Aplysia. Adv Second Messenger Phosphoprotein Res 29:529-544.

Bailey CH, Giustetto M, Huang YY, Hawkins RD, Kandel ER (2000) Is heterosynaptic modulation essential for stabilizing Hebbian plasticity and memory? Nat Rev Neurosci 1:11-20.

Bao JX, Kandel ER, Hawkins RH (1997) Involvement of pre- and postsynaptic mechanisms in posttetanic potentiation at Aplysia synapses. Science 275:969-973.

Bao JX, Kandel ER, Hawkins RH (1998) Involvement of presynaptic and postsynaptic mechanisms in a cellular analog of classical conditioning at Aplysia sensory-motor neuron synapses in isolated culture. J Neurosci $18: 458-466$.

Bertram R (2001) Differential filtering of two presynaptic depression mechanisms. Neural Comput 13:69-85.

Bertram R, Arnot MI, Zamponi GW (2002) Role for G protein G $\beta \gamma$ isoforms specificity in synaptic signal processing: a computational study. J Neurophysiol 87:2612-2623.

Buonomano DV (1999) Distinct functional types of associative long-term potentiation in neocortical and hippocampal pyramidal neurons. J Neurosci 19:6748-6754. 
Buonomano DV, Byrne JH (1990) Long-term synaptic changes produced by a cellular analog of classical conditioning in Aplysia. Science 249:420-423.

Buonomano DV, Merzenich MM (1995) Temporal information transformed into a spatial code by a neural network with realistic properties. Science 267:1028-1030.

Buonomano DV, Hickmott PW, Merzenich MM (1997) Context-sensitive synaptic plasticity and temporal-to-spatial transformations in hippocampal slices. Proc Natl Acad Sci USA 94:10403-10408.

Byrne JH (1982) Analysis of synaptic depression contributing to habituation of gill-withdrawal reflex in Aplysia californica. J Neurophysiol 48:431-438.

Byrne JH, Kandel ER (1996) Presynaptic facilitation revisited: state and time dependence. J Neurosci 16:425-435.

Byrne JH, Castellucci V, Kandel ER (1974) Receptive fields and response properties of mechanoreceptor neurons innervating siphon skin and mantle shelf in Aplysia. J Neurophysiol 37:1041-1064.

Byrne JH, Castellucci VF, Carew TJ, Kandel ER (1978a) Stimulusresponse relations and stability of mechanoreceptor and motor neurons mediating defensive gill-withdrawal reflex in Aplysia. J Neurophysiol 41:402-417.

Byrne JH, Castellucci VF, Kandel ER (1978b) Contribution of individual mechanoreceptor sensory neurons to defensive gill-withdrawal reflex in Aplysia. J Neurophysiol 41:418-431.

Byrne JH, Baxter DA, Buonomano DV, Cleary LJ, Eskin A, Goldsmith JR (1991) Neural and molecular bases of nonassociative and associative learning in Aplysia. Ann NY Acad Sci 627:124-149.

Byrne JH, Zwartjes R, Homayouni R, Critz SD, Eskin A (1993) Roles of second messenger pathways in neuronal plasticity and in learning and memory. Insights gained from Aplysia. Adv Second Messenger Phosphoprotein Res 27:47-108.

Carew TJ (2000) Behavioral neurobiology: the cellular organization of natural behavior. Sunderland, MA: Sinauer.

Castellucci VF, Schacher S (1990) Synaptic plasticity and behavioral modifications in the marine mollusk Aplysia. Prog Brain Res 86:105-115.

Castellucci VF, Pinsker H, Kupfermann I, Kandel ER (1970) Neuronal mechanisms of habituation and dishabituation of the gill-withdrawal reflex in Aplysia. Science 167:1745-1748.

Castro-Alamancos MA (1997) Short-term plasticity in thalamocortical pathways: cellular mechanisms and functional roles. Rev Neurosci 8:95-116.

Clatworthy AL, Walters ET (1993) Rapid amplification and facilitation of mechanosensory discharge in Aplysia by noxious stimulation. J Neurophysiol 70:1181-1194.

Cleary LJ, Byrne JH (1993) Identification and characterization of a multifunction neuron contributing to defensive arousal in Aplysia. J Neurophysiol 70:1767-1776.

Cleary LJ, Byrne JH, Frost WN (1995) Role of interneurons in defensive withdrawal reflexes in Aplysia. Learn Mem 2:133-151.

Cleary LJ, Lee WL, Byrne JH (1998) Cellular correlates of long-term sensitization in Aplysia. J Neurosci 18:5988-5998.

Cohen TE, Kaplan SW, Kandel ER, Hawkins RD (1997) A simplified preparation for relating cellular events to behavior: mechanisms contributing to habituation, dishabituation, and sensitization of the Aplysia gillwithdrawal reflex. J Neurosci 17:2886-2899.

Conrad P, Wu F, Schacher S (1999) Changes in functional glutamate receptors on a postsynaptic neuron accompany formation and maturation of an identified synapse. J Neurobiol 39:237-248.

Dale N, Kandel ER (1993) L-glutamate may be the fast excitatory transmitter of Aplysia sensory neurons. Proc Natl Acad Sci USA 90:7163-7167.

Eliot LS, Kandel ER, Siegelbaum SA, Blumenfeld H (1993) Imaging terminals of Aplysia sensory neurons demonstrates role of enhanced $\mathrm{Ca}^{2+}$ influx in presynaptic facilitation. Nature 361:634-637.

Eliot LS, Hawkins RD, Kandel ER, Schacher S (1994a) Pairing-specific, activity-dependent presynaptic facilitation at Aplysia sensory-motor neuron synapses in isolated cell culture. J Neurosci 14:368-383.

Eliot LS, Kandel ER, Hawkins RD (1994b) Modulation of spontaneous transmitter release during depression and posttetanic potentiation of Aplysia sensory-motor synapses isolated in culture. J Neurosci 14:3280-3292.

Fortune ES, Rose GJ (2001) Short-term synaptic plasticity as a temporal filter. Trends Neurosci 24:381-385.
Frost L, Kaplan SW, Cohen TE, Henzi V, Kandel ER, Hawkins RD (1997) A simplified preparation of LE and unidentified siphon sensory neurons to mediation and habituation of the Aplysia gill- and siphon-withdrawal reflex. J Neurosci 17:2900-2913.

Goldsmith JR, Byrne JH (1993) Bag cell extract inhibits tail-siphon withdrawal reflex, suppressed long-term but not short-term sensitization, and attenuates sensory-to-motor neuron synapses in Aplysia. J Neurosci $13: 1688-1700$.

Hayes RD, Byrne JH, Baxter DA (2003) Neurosimulation: tools and resources. In: The handbook of brain theory and neural networks, second edition (Arbib MA, ed), pp 776-780. Cambridge, MA: The MIT Press.

Hawkins RD, Abrams TW, Carew TJ, Kandel ER (1983) A cellular mechanism of classical conditioning in Aplysia: activity-dependent amplification of presynaptic facilitation. Science 219:400-405.

Hawkins RD, Kandel ER, Siegelbaum SA (1993) Learning to modulate transmitter release: themes and variation in synaptic plasticity. Annu Rev Neurosci 16:625-665.

Izhikevich EM, Desai NS, Walcott EC, Hoppensteadt FC (2003) Bursts as a unit of neural information: selective communication via resonance. Trends Neurosci 26:161-167.

Jiang XY, Abrams TW (1998) Use-dependent decline of paired-pulse facilitation at Aplysia sensory neuron synapses suggests a distinct vesicle pool or release mechanism. J Neurosci 18:10310-10319.

Kandel ER (2001) The molecular biology of memory storage: a dialogue between genes and synapses. Science 294:1030-1038.

Kupfermann I, Castellucci V, Pinsker H, Kandel ER (1970) Neuronal correlates of habituation and dishabituation of the gill-withdrawal reflex in Aplysia. Science 167:1743-1745.

Lin XY, Glanzman DL (1994) Long-term potentiation of Aplysia sensorimotor synapses in cell culture: regulation by postsynaptic voltage. Proc $\mathrm{R}$ Soc Lond B Biol Sci 255:113-118.

Manor Y, Nadim F (2001) Synaptic depression mediates bistability in neuronal networks with recurrent inhibitory connectivity. J Neurosci 21:9460-9470.

Markram H, Tsodyks M (1996) Redistribution of synaptic efficacy between neocortical pyramidal neurons. Nature 382:807-810.

McPherson DR, Blankenship JE (1991) Neuronal control of swimming in Aplysia brasiliana. I. Innervation of parapodial muscle by pedal ganglion motoneurons. J Neurophysiol 66:1338-1351.

Nadim F, Manor Y, Kopell N, Marder E (1999) Synaptic depression creates a switch that controls the frequency of an oscillatory circuit. Proc Natl Acad Sci USA 96:8206-8211.

Phares GA, Byrne JH (1999) Dynamic modulation of Aplysia sensorimotor EPSPs during bursts of sensory neuron action potentials. Soc Neurosci Abstr 25:1611.

Schacher S, Montarolo P, Kandel ER (1990) Selective short- and long-term effects of serotonin, small cardioactive peptide, and tetanic stimulation on sensorimotor synapses of Aplysia in culture. J Neurosci 10:3286-3294.

Schaffhausen JH, Fischer TM, Carew TJ (2001) Contribution of postsynaptic $\mathrm{Ca}^{2+}$ to the induction of post-tetanic potentiation in the neural circuit for siphon withdrawal in Aplysia. J Neurosci 21:1739-1749.

Scholz KS, Byrne JH (1987) Long-term sensitization in Aplysia: biophysical correlates in tail sensory neurons. Science 235:685-687.

Selig DK, Nicoll RA, Malenka RC (1999) Hippocampal long-term potentiation preserves the fidelity of postsynaptic responses to presynaptic bursts. J Neurosci 19:1236-1246.

Stopfer M, Carew TJ (1996) Heterosynaptic facilitation of tail sensory neuron synaptic transmission during habituation in tail-induced tail and siphon withdrawal reflexes of Aplysia. J Neurosci 16:4933-4948.

Sutton MA, Masters SE, Bagnall MW, Carew TJ (2001) Molecular mechanisms underlying a unique intermediate phase of memory in Aplysia. Neuron 31:143-154.

Trudeau LE, Castellucci VF (1992) Contribution of polysynaptic pathways in the mediation and plasticity of Aplysia gill and siphon withdrawal reflex: evidence for differential modulation. J Neurosci 12:3838-3848.

Tsodyks M, Markram H (1997) The neural code between neocortical pyramidal neurons depends on neurotransmitter release probability. Proc Natl Acad Sci USA 94:719-723.

Wainwright ML, Zhang H, Byrne JH, Cleary LJ (2002) Localized neuronal 
outgrowth induced by long-term sensitization training in Aplysia. J Neurosci 22:4132-4141.

Walters ET, Byrne JH (1983) Associative conditioning of single sensory neurons suggests a cellular mechanism for learning. Science 219:405-408.

Walters ET, Byrne JH (1984) Post-tetanic potentiation in Aplysia sensory neurons. Brain Res 293:377-380.

Walters ET, Byrne JH (1985) Long-term enhancement produced by activity-dependent modulation of Aplysia sensory neurons. J Neurosci 5:662-672.

Walters ET, Byrne JH, Carew TJ, Kandel ER (1983a) Mechanoafferent neurons innervating tail of Aplysia. I. Response properties and synaptic connections. J Neurophysiol 50:1522-1542.

Walters ET, Byrne JH, Carew TJ, Kandel ER (1983b) Mechanoafferent neu- rons innervating tail of Aplysia. II. Modulation by sensitizing stimulation. J Neurophysiol 50:1543-1559.

White JA, Ziv I, Cleary LJ, Baxter DA, Byrne JH (1993) The role of interneurons in controlling the tail-withdrawal reflex of Aplysia: a network model. J Neurophysiol 70:1777-1786.

Xu Y, Pieroni JP, Cleary LJ, Byrne JH (1995) Modulation of an inhibitory interneuron in the neural circuitry for the tail withdrawal reflex of Aplysia. J Neurophysiol 73:1313-1318.

Zhao Y, Klein M (2002) Modulation of the readily releasable pool of transmitter and of excitation-secretion coupling by activity and by serotonin at Aplysia sensorimotor synapses in culture. J Neurosci 22:10671-10679.

Ziv I, Baxter DA, Byrne JH (1994) Simulator for neural networks and action potentials: description and application. J Neurophysiol 71:294-308. 